\title{
Principal Components Tracking Algorithms for Synchronization and Channel Identification in UWB systems
}

\author{
J. Zhang, T. D. Abhayapala, R. A. Kennedy \\ National ICT, Australia and \\ Department of Telecommunications Engineering \\ Australian National University, Canberra ACT 0200, Australia \\ Email: jian@syseng.anu.edu.au
}

\begin{abstract}
In this paper, we investigate the reduced rank shift invariant techniques in the application of synchronization and channel identification of UWB signals. The proposed reduced rank techniques can track the principal components automatically and reduce the computational complexity significantly by transforming the generalized eigen-problem in an original high dimensional space to a lower dimensional space depending on the number of desired principal signals. Technical details in the application, including the operations of sampling, fast Fourier transform (FFT) and the capture of synchronization delay, are given. Experiments show the performance is only slightly inferior to the general full rank algorithms.
\end{abstract}

shift invariant techniques, UWB, synchronization, channel estimation

\section{INTRODUCTION}

Ultra Wideband (UWB) signals have very high resolution ability. This implies a frequency-selective channel with rich multipath in practice. Identifying and utilizing these multipath is a must for achieving satisfactory performance in a UWB receiver.

Some related research based on the traditional correlator techniques have been reported [1], [2]. The correlatorbased techniques are simple, but they only have limited resolution ability (depending on the number of samples for non-overlapped multipath signals), and are vulnerable to the intersymbol interference (ISI). In [3], a frequency approach is introduced based on the subspace methods. Although this scheme is derived from the authors' preceding work on the "sampling signals with finite rate of innovation", it is essentially same to those [4], [5] based on the well-known shift invariant techniques [6], [7].

Shift Invariant techniques, such as ESPRIT and its variants, Matrix Pencil methods and State-Space methods, are a class of signal subspace approaches with high resolution ability but relatively high computational complexity associated with the singular value decomposition (SVD) and generalized eigenvalue decomposition (GED). To make the algorithms noisestable, truncated data matrices are generally formed using SVD, and the original GED in a larger space is transformed into that in a smaller space. So rank reduction is inherent in shift invariant techniques. However, in the literature, the rank reduction is only limited to separating the signal subspace and noise subspace and the reduced rank is constrained to $L$, the number of signal sources, which is usually required to be known as a prior or to be estimated on-line.

In this paper, we present some novel joint channel identification and synchronization methods for UWB based on reduced-rank shift invariant techniques. Unlike general subspace methods, our schemes remove the constraint on $L$, and $p$ multipath signals with largest energy can be automatically tracked and identified, while the complexity is only roughly related to $p$ and can be significantly reduced. The value of $p$ can be adjusted freely to meet different performance requirements of synchronization and specific multiple-finger receivers like RAKE. In regard to the anti-aliasing filter used before sampling in [3], we will discuss the possibility of omitting it in the receiver.

The following notation is used. Matrices and vectors are denoted by boldface upper-case and lower-case letters, respectively. The conjugate transpose of a vector or matrix is denoted by the superscript $(\cdot)^{*}$, the transpose is denoted by $(\cdot)^{T}$, and the pseudo-inverse of a matrix is denoted by $(\cdot)^{\dagger}$. Finally, I denotes the identity matrix and $\operatorname{diag}(\cdots)$ denotes a diagonal matrix.

\section{REDUCED-RANK IDENTIFICATION OF PRINCIPAL COMPONENTS}

Typical harmonic retrieval problems can be addressed as the identification of unknown variables from the following equations

$$
x(k)=\sum_{\ell=1}^{L} a_{\ell} e^{j k \omega_{\ell}}+n(k), k \in[1, K]
$$

where $x(k)$ are the measured samples, $n(k)$ are the noise samples, $a_{\ell}$ and $\omega_{\ell} \in(0,2 \pi)$ are the unknown amplitudes and frequencies, to be determined.

Organize these measured samples $x(k)$ into a Hankel matrix $\mathbf{X}$ where the entries along the anti-diagonals are equal to each other, we get

$$
\mathbf{X}=\left(\begin{array}{cccc}
x(2) & x(3) & \cdots & x(Q+1) \\
x(3) & x(4) & \cdots & x(Q+2) \\
\vdots & \vdots & \ddots & \vdots \\
x(M+1) & x(M+2) & \cdots & x(K)
\end{array}\right)
$$


where $\min (M, Q) \geq L$ and $M+Q-1=K$. Without loss of generality, we assume $M \geq Q$. In the noise-free case, $\mathbf{X}$ can be factorized as

$$
\mathbf{X}=\mathbf{F}_{M} \mathbf{A} \mathbf{F}_{Q}^{T}
$$

where

$$
\begin{aligned}
& \mathbf{F}_{M}=\mathbf{F}(M), \mathbf{F}_{Q}=\mathbf{F}(Q), \\
& \mathbf{F}(m)=\left[\mathbf{f}_{m}\left(\omega_{1}\right), \mathbf{f}_{m}\left(\omega_{2}\right), \cdots, \mathbf{f}_{m}\left(\omega_{\ell}\right), \cdots, \mathbf{f}_{m}\left(\omega_{L}\right)\right], \\
& \mathbf{f}_{m}\left(\omega_{\ell}\right)=\left[e^{j \omega_{\ell}}, e^{j 2 \omega_{\ell}}, \cdots, e^{j m \omega_{\ell}}\right]^{T}, \\
& \mathbf{A}=\operatorname{diag}\left(a_{1}, a_{2}, \cdots, a_{L}\right) .
\end{aligned}
$$

The Vandermonde matrix $\mathbf{F}(m)$ exhibits the so-called shift invariant property, that is,

$$
\mathbf{F}(m)^{\uparrow d}=\mathbf{F}(m)_{\downarrow d} \boldsymbol{\Phi}^{d}
$$

where $(\cdot)^{\uparrow d}$ and $(\cdot)_{\downarrow d}$ denote the operations of omitting the first $d$ and the last $d$ rows of a matrix, respectively, and $\boldsymbol{\Phi}=$ $\operatorname{diag}\left(e^{j \omega_{1}}, e^{j \omega_{2}}, \cdots, e^{j \omega_{L}}\right)$ contains the desired frequencies. This property facilitates the development of various shift invariant techniques. By constructing two $L$ rank matrices $\mathbf{Y}_{1}$ and $\mathbf{Y}_{2}$ with inherent shift invariant property, the diagonal elements of $\Phi$ can be obtained by solving the generalized eigenvalues of the matrix pencil $\left\{\mathbf{Y}_{1}-\xi \mathbf{Y}_{2}\right\}$. These two matrices $\mathbf{Y}_{1}$ and $\mathbf{Y}_{2}$ can be constructed directly from $\mathbf{X}$ using $\mathbf{Y}_{1}=\mathbf{X}_{\downarrow d}$ and $\mathbf{Y}_{2}=\mathbf{X}^{\uparrow d}$, or from the correlation matrices of $\mathbf{X}$, or from the singular vectors of $\mathbf{X}$. The use of $d>1$ can improve resolution ability and result to smaller variance of estimates [8], but $d$ must be chosen to ensure $d<2 \pi / \max \left(\omega_{\ell}\right)$ in order to avoid phase ambiguities.

\section{A. Principal subspace and frequency estimation}

Suppose that the constructed $\mathbf{Y}_{1}$ and $\mathbf{Y}_{2}$ are $(M-d) \times Q$ noise-free matrices. Since $\mathbf{Y}_{1}$ has rank $L$, the compact SVD of $\mathbf{Y}_{1}$ has the form

$$
\begin{aligned}
\mathbf{Y}_{1}=\mathbf{U} \boldsymbol{\Lambda} \mathbf{V}^{*} & =\left[\mathbf{U}_{p} \mathbf{U}_{r}\right]\left[\begin{array}{cc}
\boldsymbol{\Lambda}_{p} & \mathbf{0} \\
\mathbf{0} & \boldsymbol{\Lambda}_{r}
\end{array}\right]\left[\begin{array}{ll}
\mathbf{V}_{p} & \mathbf{V}_{r}
\end{array}\right]^{*} \\
& =\mathbf{U}_{p} \boldsymbol{\Lambda}_{p} \mathbf{V}_{p}^{*}+\mathbf{U}_{r} \boldsymbol{\Lambda}_{r} \mathbf{V}_{r}^{*}
\end{aligned}
$$

where the $L \times L$ diagonal matrix $\Lambda$ contains singular values in descending order, the $(M-d) \times L$ matrix $\mathbf{U}$ and $Q \times L$ matrix $\mathrm{V}$ consist of left and right singular vectors, respectively. $\mathbf{U}_{p}$ $\left(\mathbf{V}_{p}\right)$ and $\mathbf{U}_{r}\left(\mathbf{V}_{r}\right)$ are the left and right submatrices of $\mathbf{U}$ $(\mathbf{V})$, associated with the $p$ principal and the remaining $L-p$ smaller singular values, respectively.

Multiply the matrix pencil $\left(\mathbf{Y}_{1}-\xi \mathbf{Y}_{2}\right)$ by $\mathbf{U}_{p}^{*}$ from the left and by $\mathbf{V}_{p}$ from the right, we get a new $p \times p$ matrix pencil

$$
\left(\boldsymbol{\Lambda}_{p}-\xi \mathbf{U}_{p}^{*} \mathbf{Y}_{2} \mathbf{V}_{p}\right),
$$

where we have utilized the orthogonality between the columns of $\mathbf{U}_{p}$ and $\mathbf{U}_{r}$, and $\mathbf{V}_{p}$ and $\mathbf{V}_{r}$.

For the new matrix pencil, we have the following results.

Proposition 1: The matrix pencil $\left(\boldsymbol{\Lambda}_{p}-\xi \mathbf{U}_{p}^{*} \mathbf{Y}_{2} \mathbf{V}_{p}\right)$ has $p$ distinctive generalized eigenvalues $\xi_{\ell}, \ell=1,2, \cdots, p$, and in most cases, the angles of $\xi_{\ell}$ are 1) equal or 2) good approximations to the $p$ frequencies $\omega_{\ell}$ up to a known scalar, corresponding to $p$ harmonics with largest energy.
Proof: The proof is given in [9].

Substituting estimated frequencies into (1), the amplitudes $a_{\ell}$ can be obtained by solving a Vandermonde system using least squares type algorithms [10]. However, in the case when only $p$ out of $L$ frequencies are known, the estimates of $p$ amplitudes obtained by solving under-determined linear equations of (1) will comprise large error. Alternatively, when $\mathbf{Y}_{1}$ and $\mathbf{Y}_{2}$ are formed as the correlation matrices of $x(k)$, for example,

$$
\begin{aligned}
\mathbf{Y}_{1} & =\mathbf{X}_{\downarrow d}\left(\mathbf{X}_{\downarrow d}\right)^{*}, \\
\mathbf{Y}_{2} & =\mathbf{X}^{\uparrow d}\left(\mathbf{X}_{\downarrow d}\right)^{*},
\end{aligned}
$$

the energy of the harmonics can be estimated in a subspace method according to the following proposition.

Proposition 2: When $\mathbf{Y}_{1}$ and $\mathbf{Y}_{2}$ are constructed similar to (8), the energy of every harmonic, $\left|a_{\ell}\right|^{2}$, can be well approximated by

$$
\left|a_{\ell}\right|^{2} \approx \frac{\boldsymbol{\theta}_{\ell}^{*} \boldsymbol{\Lambda}_{p} \boldsymbol{\theta}_{\ell}}{\left|\boldsymbol{\theta}_{\ell}^{*} \mathbf{U}_{p} \mathbf{f}_{M-d}\left(\omega_{\ell}\right)\right|^{2}},
$$

where $\boldsymbol{\theta}_{\ell}$ is the generalized eigenvector corresponding to the generalized eigenvalue $\xi_{\ell}$ (and then frequency $\omega_{\ell}$ ).

Proof: The proof is given in [9].

A necessary condition for the above proposition is that the product $\mathbf{F}_{Q}^{T}\left(\mathbf{F}_{Q}^{T}\right)^{*}$ need resemble an identity matrix. This condition can only be met when $Q$ is larger enough and there is no frequency close to zero or $\pi$.

In [9], we will show there is another method in which the condition can be waived by constructing a Hermitian data matrix.

\section{B. Fast Algorithms}

Since only $p$ out of $L$ principal singular values and vectors are required, the computation can be simplified by applying fast algorithms with lower complexity, such as the power method [11]. For each dominant singular value and vector, the power method has a computational order of $(M-d)^{2}$ for a $(M-d) \times(M-d)$ Hermitian matrix. To be noted, in the power method, the speed of convergence is highly related to the ratio between the two principal singular values of the matrix. The larger the ratio is, the faster it converges. For the series of principal singular values, several iterations can usually achieve convergence.

For a Hermitian matrix $\mathbf{Y}_{1}$, the power method generates $p$ principal singular values and vectors as follows:

1) Let $i=1$;

2) Generate the dominant eigenvalue $\lambda_{i}$ and eigenvector $\mathbf{v}_{i}$ of $\mathbf{Y}_{1}$ using the power method;

3) Using the deflation operation to update $\mathbf{Y}_{1}$ : $\mathbf{Y}_{1}=\mathbf{Y}_{1}-\lambda_{i} \mathbf{v}_{i} \mathbf{v}_{i}^{*}$

4) Let $i=i+1$, and repeat 2 until $i=p+1$.

When $\mathbf{Y}_{1}$ is not a symmetric or Hermitian matrix, a similar algorithm is applicable in which the left and right singular vectors should be generated by constructing $\mathbf{Y}_{1} \mathbf{Y}_{1}^{*}$ and $\mathbf{Y}_{1}^{*} \mathbf{Y}_{1}$, respectively. 


\section{JOINT SYNCHRONIZATION AND CHANNEL IDENTIFICATION}

We consider a general transmitted UWB signal $s(t)$ in a single user system in this paper. The signal $s(t)$ could be a spread spectrum (SS) signal (e.g., time hopping or direct sequence hopping) or non-SS signal (e.g., single pulse), but it should be unmodulated or modulated with identical known data. We assume the spread spectrum sequence is known in a SS system. A simplified time delayed line (TDL) multipath model is adopted. The TDL model can be represented as

$$
h(t)=\sum_{\ell=1}^{L} a_{\ell} \delta\left(t-\tau_{\ell}\right)
$$

where $\tau_{\ell}$ is the $\ell$ th multipath delay, $a_{\ell}$ is the $\ell$ th multipath gain with phase uniformly set to $\{ \pm 1\}$.

When a symbol sequence $\left\{s_{i}(t)\right\}$ are transmitted over this channel, the received signal $r(t)$ is

$$
r(t)=\sum_{i} \sum_{\ell=1}^{L} a_{\ell} s_{i}\left(t-i T_{s}-\tau-\tau_{\ell}\right)+n(t),
$$

where $\tau$ is the synchronization delay between the receiver and the transmitter, $T_{s}$ is the symbol period, $n(t)$ represents addition white Gaussian noise (AWGN) with mean zero and variance $\sigma_{0}^{2}$.

To set up the connection between (11) and (1), we can transform (11) from time domain to frequency domain. Considering the efficiency, Discrete Fourier Transform (DFT) can be applied to the samples of $r(t)$. However, when applying the DFT, some technical details should be dealt with carefully.

\section{A. Sampling of Signals}

Since the system is not synchronized yet, whatever the signal $s(t)$ is, the width of the sampling window should equal the integral times of the symbol period and be larger than the maximal multipath spread. Assume that the sampling period is $T$, the number of samples is $K_{1}$, and the obtained samples from (11) are $\{r(m)\}$, where $m \in\left[1, K_{1}\right]$. Two scenarios need be considered for the DFT coefficients.

1) Sampling of Widely Separated Pulses: When the interval between the continuously transmitted pulses is larger than the maximum spread of the multipath, ISI does not exist in the samples. Let the sampling length $T K_{1}$ equal the symbol period $T_{s},\{s(m)\}$ be the samples of $s_{i}(t)$, and $\{n(m)\}$ be the samples of the noise $n(t)$, then the DFT coefficients of (11) can be represented as

$$
R(k)=S(k) \sum_{\ell=1}^{L} a_{\ell} e^{-j k \Omega_{0}\left(\tau+\tau_{\ell}\right)}+N(k), k \in\left[1, K_{1}\right],
$$

where $\Omega_{0}=2 \pi /\left(T K_{1}\right)$ is the basic frequency, $S(k)$ and $N(k)$ are the DFT coefficients of $\{s(m)\}$ and $\{n(m)\}$, respectively.
2) Sampling of Closely Spaced Pulses: When the interval between the transmitted pulses is smaller than the maximum spread of the multipath, ISI is generated. Assume that at most $\Delta i$ symbols can cover the multipath spread. Represent the $\Delta i$ symbols as

$$
s_{\Delta i}(t)=\sum_{i=i_{1}}^{i_{1}+\Delta i-1} s_{i}\left(t-i T_{s}\right)
$$

where $i_{1}$ is the index of any symbol, and let $\{s(m)\}, m \in$ $\left[1, K_{1}\right]$ be the samples of $s_{\Delta i}$. When symbols are transmitted continuously without interruption, it can be shown that the Circular Shift Property of the DFT enables the measured samples in the frequency domain to be ISI-free, and (12) is also satisfied in this case.

3) Influence of Sampling period on the Resolution Ability: From the expression of the circular shift property, it seems that the delays are implicitly aligned to the integral multiples of the sampling period. Actually, this is not necessary when the signal is bandlimited and it is sampled above the Nyquist rate. According to the sampling theory, any bandlimited signal can be reconstructed without distortion when sampled above the Nyquist rate, no matter when the sampling starts.

On the contrary, if the signal is not bandlimited or it is sampled below the Nyquist rate, aliasing will affect the accuracy of estimates due to the loss of the circular shift property between the DFT coefficients of original signals and their time shifted versions. Besides, the jitter of sampling epoch will also cause slight errors. In this sense, the sampling period largely determines the accuracy of estimates.

However, interestingly, in the delay tracking problem, if the delays can be translated into integral times of the sampling period, aliasing will not influence the estimation at all. Intuitively, the delay tracking now is equivalently operated over some discrete samples which are predetermined and fixed. This result seems to suggest that a resolution of the estimates up to half of the sampling period is always achievable no matter how severe the aliasing degree is.

\section{B. Spectrum aliasing and DFT coefficients}

A key problem in DFT is the aliasing arisen by sampling below the Nyquist rate. Due to the ultra wide bandwidth of UWB signals, sampling rate usually can not be high enough to avoid aliasing in practical implementations. In this case, the received signal can be passed through a band-limited filter (called as anti-aliasing filter) before sampling as suggested in [3]. However, this filter should be an analog filter but not a digital one. Otherwise higher sampling frequency in the digital filter will be required to avoid the distortion of a desired spectrum. The disadvantage with such an analog device in a low-power UWB system is obvious. Below, we will see that this filter is not necessary when we check the property of the Fourier transform of Gaussian monocycles.

The spectrum of $s_{\Delta i}$ can be expressed as the sum of several shifted versions of the spectrum of the basic monocycle. The degree and range of spectrum aliasing in the DFT coefficients of $s_{\Delta i}$ are largely determined by the aliasing of the basic monocycle. Then, by observing the aliasing in the DFT of the 
basic monocycle, we can choose those DFT coefficients with negligible errors.

On the other hand, DFT coefficients with larger energy should be chosen to avoid blowing up the noise in the de-convolution operation. When the signal $s_{\Delta i}$ consists of multiple monocycles, the signal spectrum deviates largely from the spectrum of the basic monocycle. So the selection of DFT coefficients varies from time to time and has to be determined on-line.

When strong narrow-band interference is present and the interference spectrum is known, the interference can be readily removed by selecting those coefficients in the unaffected spectrum.

\section{Application of the principal components tracking algorithm}

De-convolution is defined as the operation of dividing $R(k)$ by $S(k)$ in (12), the reverse of convolution viewed in the frequency domain. After the de-convolution operation, we get an expression identical to the harmonic retrieval model in (1). Then the synchronization and channel identification algorithm can be summarized as follows:

1) Sampling the received signal using period $T$ in a window with width $T K_{1}$. Make sure $T K_{1}$ equals integer times of the symbol period $T_{s}$ and be larger than the maximal multipath spread;

2) Applying FFT to the samples and select $K$ DFT coefficients carefully;

3) After de-convolution, forming Hankel data matrix X, and using principal components tracking algorithms to estimate the $p$ delays with largest energy (sum of $\tau$ and $\tau_{\ell}$ ). (If the fading amplitudes $a_{\ell}$ are required, correlation matrices should be used)

4) Resolve $\tau$ and $\tau_{\ell}$ from the estimated delays.

The last step is necessary since the estimated delays in step (3) are the sums between the synchronization delay and every multipath delays. There is a phase-ambiguity problem with these sums as the delays may become circularly shifted. This could happen when sampling starts in the middle of multipath delays. Our solution is first to make $T K_{1}$ be much larger than the maximal multipath delay, then separate $\tau$ and $\tau_{\ell}$ according to the following criteria:

- Sorting the estimates in ascending order, $\left\{\hat{\tau}_{1}, \hat{\tau}_{2}, \cdots, \hat{\tau}_{p}\right\}$. If the gap between two adjoining estimates is larger than a threshold $\tau_{\text {th }}$, for example, $\hat{\tau}_{p_{1}}-\hat{\tau}_{p_{1}-1}>\tau_{\text {th }}$, then $\hat{\tau}_{p_{1}}$ equals the sum of the synchronization delay and the first desired multipath. And all the estimates need be updated to

$$
\left\{\hat{\tau}_{p_{1}}, \hat{\tau}_{p_{1}+1}, \cdots, \hat{\tau}_{p}, \hat{\tau}_{1}+T K_{1}, \cdots, \hat{\tau}_{p_{1}-1}+T K_{1}\right\},
$$

that is, the original $\hat{\tau}_{1}, \cdots, \hat{\tau}_{p_{1}-1}$ are updated by adding $T K_{1}$ to themselves. Now, the receiver can synchronize to the multipath with delay $\hat{\tau}_{p_{1}}$ which implicity assumes the delay of the first multipath of interest is zero. Then the difference between the updated estimates and the first desired multipath are the relative multipath delays.

- Otherwise, the smallest estimate is the first multipath of interest and no update is needed.
This judgement is based on the assumption that the gap between any two multipath is smaller than the threshold $\tau_{\text {th }}$, which is generally close to the difference between the window width of the sampling $T K_{1}$ and the maximal mltipath delay. In practice, the multipath components with larger energy usually have smaller delays, so the threshold need not be very large.

\section{Complexity of Our Scheme}

The complexity of our algorithm depends on the required resolution ability and performance of estimation. The resolution ability is roughly determined by the sampling period. The smaller the sampling period is, the higher the resolution ability is. The performance of estimation is mainly influenced by the signal to noise ratio (SNR), and the dimension of the matrices $\mathbf{Y}_{1}$ and $\mathbf{Y}_{2}$. Then the sampling period is the key parameter in both the complexity and performance since the main computation cost of our algorithm is associated with FFT and GED. For an $K_{1}$-point FFT $^{1}$, the computational workload is $K_{1}\left(3 \log _{2} K_{1}-1\right) / 2$ when using a Cooley-Tukey radix-2 algorithm. Plus the complexity of the power method and the GED of a $p \times p$ matrix, the total complexity of our reduced rank algorithms is in the order of $K_{1} \log _{2} K_{1}+K_{1}^{2}+p^{3}$, while the complexity of general shift invariant techniques is in the order of $K_{1} \log _{2} K_{1}+K_{1}^{3}+L^{3}$.

\section{Simulations}

The second order Gaussian monocycle $p(t)$ is used as the basic pulse

$$
p(t)=\left[1-4 \pi\left(\left(t-t_{p}\right) / t_{p}\right)^{2}\right] \exp \left(-2 \pi\left(\left(t-t_{p}\right) / t_{p}\right)^{2}\right),
$$

where $t_{p}$ parameterizes the effective pulse width. To generalize the analysis, $t$ is normalized with respect to $t_{p}$.

Fig. 1 depicts how the magnitude of DFT coefficients and the degree of aliasing change when a sequence of Gaussian monocycles are transmitted over a TDL multipath channel. The transmitted signal consists of two of the pulse $p(t)$ separated by $4 t_{p}$. The multipath channel simply consists of two tags with delays $2.27 t_{p}, 4.41 t_{p}$ and amplitudes $0.85,0.51$, respectively. The sampling period is $T=0.3 t_{p}$ (roughly six samples per pulse). From the figure, we can see the degree of aliasing changes, and is less serious than that in the single pulse case. From the statistical viewpoint, this is always the case due to the cancellation between different delayed versions of the monocycle.

To test the performance of our algorithms in practical implementations, we use the channel model proposed in [12] by IEEE 802.15.3a. The channel impulse response (CIR) is reproduced using $t_{p}=1$ for the line-of-sight model (CM1). The transmitted signal is a single pulse $p(t)$. The first $L=50$ multipath signals in each of 20 CIRs are truncated as inputs. The multipath signal is sampled with period $T=0.3 t_{p}$ The rooted mean squared error (RMSE) of the multipath delay estimates averaged over 10 realizations for each CIR is shown in Fig. 2. In the experiments, the ratio of successfully tracking the multipath with largest energy is about $80 \%$. The

\footnotetext{
${ }^{1} K_{1}$ need equal a power of 2 , or the zero padding is required.
} 


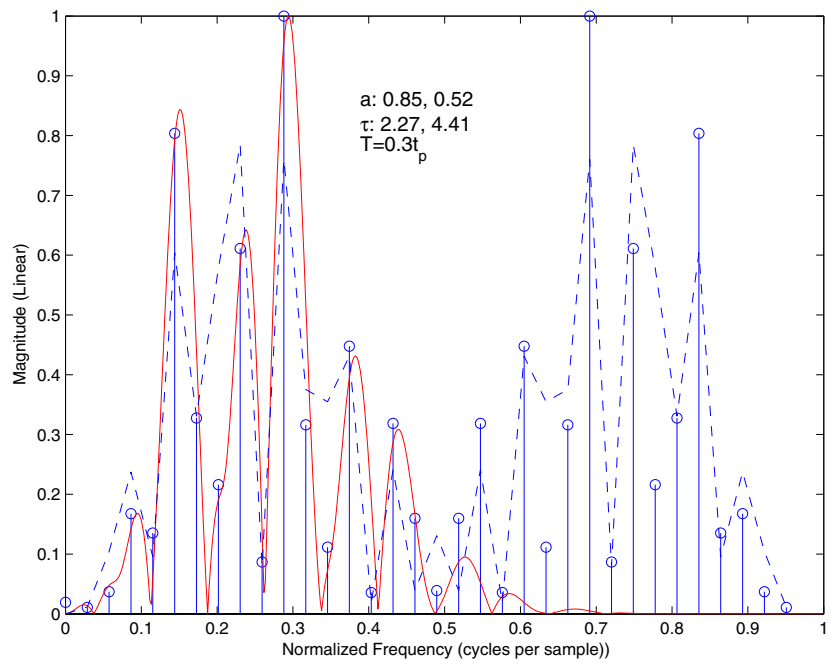

Fig. 1. Illustration of DFT coefficients and the aliasing for a sequence of Gaussian Monocycles. Solid curve: CFT of the received signal; Dashed curve: DFT of the transmitted signal; Stems with circles in the end: DFT of the received signal.

ratio of "hit" is influenced by the overlapping of multipath components, the burst of noise and the limitation of the algorithm itself. The Mean Squared Error shown in Fig. 2 is the statistical result with wrong "hit" excluded. In the figure, the solid lines correspond to $p=10$, and the dashed lines correspond to $p=L=50$. The two curves without marks depict the performance of the practical implementations (Sampling, FFT and identification). As comparisons, the two curves marked with circles depict the result from the harmonics model when the multipath coefficients are directly fed into (1). The comparisons show that the loss of performance is significant during the sampling, FFT and de-convolution operations. This loss is less dependent of the sampling period according to our experiments. Even when the sampling period is as small as $0.1 t_{p}$ and the aliasing is almost invisible, the RMSEs are little improved compared to those shown in Fig. 2. The possible reasons are that 1) the estimation errors of the multipath delays are enlarged by a scalar of $T K_{1}$ since the directly estimated parameters are frequencies and 2) the sampling operation partly determines the resolution ability according to the FFT because the shift property of DFT requires the time shifts to be aligned to the integral times of the sampling period $T$. This resolution can not be improved any longer in the posterior operations. Actually, in the experiments, we find the RMSEs corresponding to the $10 \mathrm{~dB}$ SNR are already very close to the RMSEs in the noise-free case.

The performance for closely spaced pulse are similar to that in the single-pulse case. Due to space limitation, the results are not listed here.

\section{CONCLUSION}

To reduce the complexity of general subspace-based delay estimation algorithms, we introduce the reduced rank shift invariant techniques which can track the principal components automatically. Experiments show that our improved algorithm can achieve the performance slightly inferior to that by the

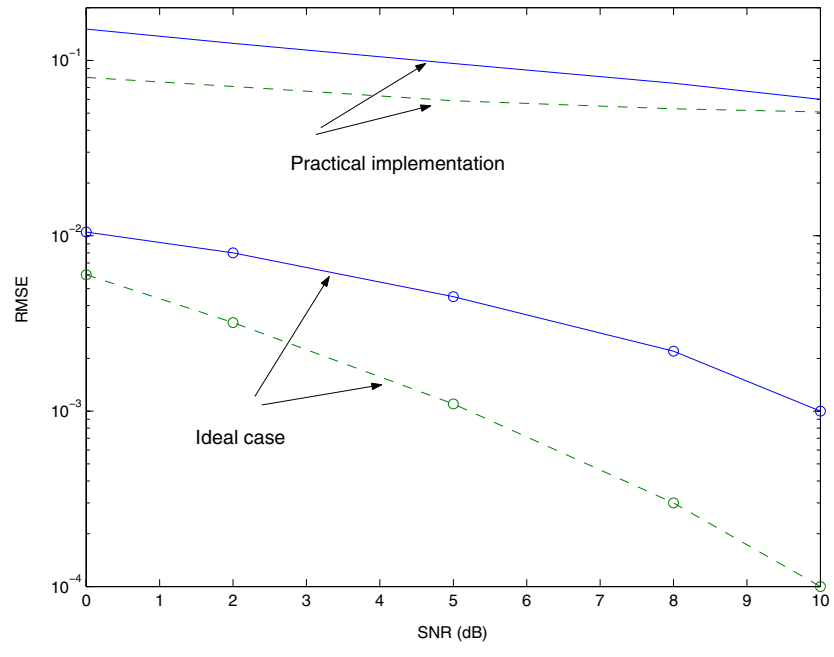

Fig. 2. Normalized RMSE of the multipath delay estimates vs the SNR. Solid lines: $p=10$; dashed lines: $p=L=50$.

full rank algorithm, but with significantly reduced complexity. However, both algorithms exhibit less attractive performance in practical implementations in UWB systems due to the inherent limitations on the resolution ability. A better scheme seems to combine these shift invariant techniques in a two-staged algorithm. The shift invariant techniques are used in the first stage to determine parameters quickly and roughly, and the second stage acts as a refinement process where the searchingbased Maximum Likelihood algorithm or a correlator with dense samples could be applied.

\section{REFERENCES}

[1] E. A. Homier and R. A. Scholtz, "Rapid acquisition of Ultra-Wideband signals in the dense multipath channel," in Proc. IEEE Conf. on UWB Systems and Technologies (UWBST), May 2002, pp. 105 - 109.

2] V. Lottici, A. D'Andrea, and U. Mengali, "Channel estimation for UltraWideband communications," IEEE J. Select. Areas Commun., vol. 20(9), pp. $1638-1644$, Dec. 2002.

[3] I. Maravic and M. Vetterli, "Low-complexity subspace methods for channel estimation and synchronization in Ultra-Wideband systems," in Proc. International Workshop on UWB Systems, Oulu, Finland, June 2003.

[4] A. J. van der Veen, M. C. Vanderveen, and A. Paulraj, "Joint angle and delay estimation using shift-invariance techniques," IEEE Trans. Signal Processing, vol. 46(2), pp. 405-418, Feb. 1998.

[5] A. L. Swindlehurst, "Time delay and spatial signature estimation using known asynchronous signals," IEEE Trans. Signal Processing, vol. 46(2), pp. 449-462, Feb. 1998

[6] B. D. Rao and K. S. Arun, "Model based processing of signals: A state space approach," Proc. IEEE, vol. 80(2), pp. 283-309, Feb. 1992.

[7] A. J. van der Veen, E. F. Deprettere, and A. L. Swindlehurst, "Subspacebased signal analysis using singular value decomposition," Proc. IEEE, vol. 81(9), pp. 1277-1308, Sep. 1993.

[8] M. Goldburg and R. Roy, "Application of ESPRIT to parameter estimation from uniformly sampled data," in Proc. IEEE ICASSP, Albuquerque, NM, 1990.

[9] J. Zhang, R. A. Kennedy, and T. D. Abhayapala, "Joint synchronization and channel identification in UWB systems using shift invariant techniques," IEEE Trans. Commun., May 2004, submitted for publication.

[10] L. L. Scharf, Statistical Signal Processing: Detection, Estimation, and Time Series Analysis, Addison-Wesley Publishing Company, New York, 1991.

[11] G. H. Golub and C. F. Van Loan, Matrix Computations, The Johns Hopkins University Press, Baltimore and London, third edition, 1996.

[12] J. Foerster et al., "Channel modeling sub-committee report final," IEEE P802.15 Working Group for Wireless Personal Area Networks (WPANs), IEEE P802.15-02/490r1-SG3a, Feb. 2003. 